\title{
Enhancing the efficacy of innate immune agonists: could nanolipoprotein particles hold the key?
}

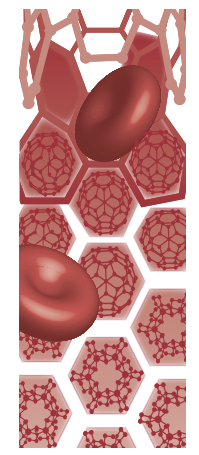

\author{
"Modulation of the innate immune system for infectious disease has the potential \\ for truly broad-spectrum efficacy...
}

Keywords: agonist $\bullet$ immune modulation • infectious disease $\bullet$ innate immunity

- nanolipoprotein particles $\bullet$ nanotechnology

\section{Innate immune modulation for infectious disease}

Immune modulation strategies have been applied extensively in cancer therapeutics for several decades with varied success. Applying similar strategies to prevent or treat infectious disease has transformative potential and remains poorly explored. Modulation of the innate immune system for infectious disease has the potential for truly broad-spectrum efficacy due to the highly conserved nature of the system and its co-evolution with pathogens over millennia [1], such that disparate pathogens utilize similar host innate immune pathways during the infectious process. Therefore, targeting of these pathways offers an attractive, alternative broad-spectrum approach for infectious disease therapeutics. Along these lines, several studies have elegantly demonstrated that prophylactic and postexposure methodologies involving innate immune stimulation prevent or ameliorate infections in animal models [2-9]. The concept of immune modulation as a strategy for infectious diseases relevant for public health and biosecurity has been reviewed over the last several years by a number of groups, which noted that a countermeasure strategy targeting the host and not the pathogen directly might greatly reduce the emergence of antibiotic resistance and may prove to be efficacious in special populations for which antibiotic and vaccination therapies are not optimal $[10,11]$.

Our knowledge of how innate immune responses are initiated, driven and maintained during infection, coupled with our knowledge of those key innate immune cells, receptors and signaling pathways involved, has opened the possibility for the rational design of targeted host-based therapeutics. Toll-like receptors (TLRs), a key class of pattern recognition receptors, are integral in initiating the immune response to infection $[1,12]$. Furthermore, robust TLR activation is a key driver in sustaining an adaptive immune response, as well as eventual pathogen clearance [1,12]. When coupled with their well-documented association with myriad infectious diseases, the importance of TLRs as therapeutic targets is widely accepted. While TLR agonists have been extensively explored as vaccine adjuvants, their use in TLR monotherapy to induce innate immune activation has been less thoroughly explored to enhance host resistance to infection by a variety of pathogens [2-9] and represents a potential paradigm shift in our approach to combat infectious diseases.

\section{Drawbacks of current approaches}

To date, the clinical potential of TLR agonists has not been fully realized despite these compounds being rigorously evaluated in numerous clinical trials for a variety of indications. While more progress has been made toward the clinical translation of TLR agonists as vaccine adjuvants rather than as standalone therapeutics, significant limitations exist for both applications [12,13]. With respect to TLR monotherapy, significant obstacles for the widespread application of this approach need to be addressed in order to facilitate clinical translatability. First, studies
Nicholas O Fischer

Biosciences \& Biotechnology Division, Lawrence Livermore National Laboratory, Livermore, CA 94550, USA

Craig Blanchette

Biosciences \& Biotechnology Division, Lawrence Livermore National Laboratory, Livermore, CA 94550, USA

Amy Rasley Author for correspondence: Biosciences \& Biotechnology Division, Lawrence Livermore National Laboratory, Livermore, CA 94550, USA rasley2@|Inl.gov

Future $\because$
Medicine $\%$ part of 
using TLR agonists to prevent infection demonstrate only marginal and short-lived protection, severely limiting their utility in both pre- and post-exposure scenarios. Second, high doses are often required to afford protection in vivo raising substantial toxicity concerns. Finally, TLR agonists tend to exhibit poor biodistribution and pharmacokinetic profiles and poor cellular uptake in vivo, thereby limiting their bioavailability and efficacy $[12,13]$. Hence, innovative strategies to improve the therapeutic index of TLR agonists in vivo are urgently needed in order to fully realize and maximize their potential for infectious disease treatment and/or prevention for both adjuvants and monotherapy applications.

\section{Nanoparticle delivery of innate immune agonists}

Nanoparticle platforms have recently been developed as tools to more efficiently deliver a variety of therapeutics, including drugs, vaccines and imaging agents [14]. It has been widely demonstrated that delivery of therapeutics via a nanoparticle platform can overcome many of the limitations of conventional administration, including nonspecific biodistribution and targeting, poor aqueous solubility, limited oral bioavailability and low therapeutic indices [15]. Based on these previous findings, we believe that successful treatment of infectious diseases through immune modulation strategies targeting TLRs may benefit significantly from the use of nanoparticle platforms for the delivery of TLR agonists.

One particular platform that is well suited for such an application is nanolipoprotein particles (NLPs; also known as reconstituted high density lipoproteins and nanodiscs). These biomimetic nanoparticles, which are comprised of apolipoproteins and lipids, have been successfully used to address both fundamental science, as well as clinically driven applications. NLPs assume a loosely discoidal morphology, nominally featuring a lipid bilayer stabilizing the apolipoprotein. While these types of nanoparticles are primarily used for solubilizing and stabilizing membrane proteins in discrete, native lipid environments for biophysical and structural characterization [16], their utility for in vivo applications has not been neglected. Of particular note are the uses of these biomimetic nanoparticles in the delivery of therapeutic drugs, enhancing a drug's therapeutic index, diagnostic imaging and vaccine applications [17-20].

We believe this platform, in particular, is advantageous for the delivery of immune agonists because there are several features intrinsic to the NLP that are of importance to any in vivo delivery platform. First, since these particles are naturally present in the human body, the NLP platform is less likely to result in issues facing other nanoparticle systems that are currently used for the delivery of therapeutics, such as unwanted off-target effects, stability in complex biological fluids and toxicity. Second, the fundamental versatility in NLP selfassembly accommodates a plethora of starting reagents (i.e., apolipoproteins and lipidic molecules), whereby the NLP size can be tailored by both pairing of the constituents, as well as their molar ratios. Third, NLPlike particles provide multiple means of cargo conjugation or incorporation, spanning a large range of cargo molecules of disparate physicochemical characteristics. This last point cannot be overemphasized, as hydrophobic moieties can be contained within the hydrophobic core of the NLP lipid bilayer, amphipathic moieties can be anchored to the lipid bilayer, and bioconjugation of soluble molecules can be achieved by incorporating functionalized lipids bearing reactive polar headgroups. As such, the transformative potential of this class of nanoparticle as a universal platform for delivering and enhancing innate immune agonists is appealing.

$$
\begin{gathered}
\text { "[A] multiagonist approach will likely } \\
\text { ensure robust stimulation of key innate } \\
\text { immune cells and may also facilitate the } \\
\text { investigation of other pattern recognition } \\
\text { receptors demonstrated to be important in a } \\
\text { variety of diseases." }
\end{gathered}
$$

As a proof of concept, we recently examined the effect of TLR agonist delivery via the NLP platform relative to administration of the TLR agonist alone [2]. We demonstrated that conjugation of two disparate (in terms of physical and chemical characteristics) TLR agonists, MPLA and CpG, was readily achieved. Subsequent in vitro and in vivo assessment of immunostimulatory properties clearly demonstrated that conjugation of TLR agonists to NLPs improved cellular uptake and activation of key innate immune effector cells relative to administration of the TLR agonist alone. In addition, when administered to mice prior to a lethal influenza virus challenge, complete protection from weight loss-induced death was observed only when the TLR agonist was conjugated to the NLP. Similar activation profiles were also observed in primary human innate immune cells, a positive indicator for the translatability of TLR agonist monotherapy to humans. Taken together, these combined findings provide strong evidence for the potential of utilizing nanoparticle technologies in combination with TLR agonists for the treatment of infectious diseases.

\section{Future prospects}

In a natural infection, many distinct innate immune receptors contribute in concert to initiate the innate 
immune response, and current data indicate that stimulation of multiple TLRs leads to synergistic cytokine production in vivo. This variety in signaling might suggest that multiagonist formulations activating a cluster of different TLRs, may lead to highly effective protection by more closely resembling the innate immune responses observed during natural infections. The versatility in accommodating chemically distinct and structurally diverse molecules during NLP production allows for the co-localization of multiple, different TLR agonists on a single NLP, thereby allowing rapid testing of multiple TLR agonist combinations. This multiagonist approach will likely ensure robust stimulation of key innate immune cells and may also facilitate the investigation of other pattern recognition receptors demonstrated to be important in a variety of diseases.

\section{"Combining enhanced TLR therapy with traditional therapeutic modalities, such as antibiotics, is also a very promising consideration. Such a strategy may be especially useful in limiting the duration of antibiotic therapies...}

Identification and validation of enhanced innate immune modulation using NLP-based formulations would necessarily be followed by numerous important studies. Rigorous efforts to refine and optimize such formulations to minimize the potential for overstimulation and deleterious side effects is paramount, as such adverse effects would be difficult to predict a priori. Evaluating their efficacy in multiple disease models is also extremely important, given the documented dif-

\section{References}

1 Beutler B. Innate immunity: an overview. Mol. Immunol. 40(12), 845-859 (2004).

2 Weilhammer DR, Blanchette CD, Fischer NO et al. The use of nanolipoprotein particles to enhance the immunostimulatory properties of innate immune agonists against lethal influenza challenge. Biomaterials 34(38), 10305-10318 (2013).

3 Wong JP, Christopher ME, Viswanathan S et al. Antiviral role of Toll-like receptor-3 agonists against seasonal and avian influenza viruses. Curr. Pharm. Design 15(11), 1269-1274 (2009).

4 Norton EB, Clements JD, Voss TG, Cardenas-Freytag L. Prophylactic administration of bacterially derived immunomodulators improves the outcome of influenza virus infection in a murine model. J. Virol. 84(6), 2983-2995 (2010).

5 Lembo A, Pelletier M, Iyer R et al. Administration of a synthetic TLR4 agonist protects mice from pneumonic tularemia. J. Immunol. 180(11), 7574-7581 (2008).

6 Elkins KL, Rhinehart-Jones TR, Stibitz S, Conover JS, Klinman DM. Bacterial DNA containing CpG motifs ferences in sensitivities that exist between the immune response to TLR agonists among different species. Evaluation of dose and route of administration, as well as dosing regimen, are important parameters that need to be assessed for each infectious disease model. Combining enhanced TLR therapy with traditional therapeutic modalities, such as antibiotics, is also a very promising consideration. Such a strategy may be especially useful in limiting the duration of antibiotic therapies, which for many infectious agents relevant for both public health and biosecurity, is extremely prolonged. The prospect of a broad-spectrum therapy for infectious disease using innate immune modulation is extremely attractive, and platform strategies such as the NLP may hold the key by both enhancing and tailoring the innate immune response using available agonists to realize this goal.

\section{Financial \& competing interests disclosure}

Two patents were filed on utilizing the nanolipoprotein particle platform for vaccine applications: 'Nanolipoprotein particles and related compositions, methods and systems' (application number: 12469533) and 'Immunostimulatory nanoparticles and related compositions, methods and systems' (application number: 12604362). The authors have no other relevant affiliations or financial involvement with any organization or entity with a financial interest in or financial conflict with the subject matter or materials discussed in the manuscript. This includes employment, consultancies, honoraria, stock ownership or options, expert testimony, grants or patents received or pending, or royalties.

No writing assistance was utilized in the production of this manuscript.

stimulates lymphocyte-dependent protection of mice against lethal infection with intracellular bacteria. J. Immunol. 162(4), 2291-2298 (1999).

7 Jiang T, Zhao H, Li XF et al. CpG oligodeoxynucleotides protect against the $2009 \mathrm{H} 1 \mathrm{~N} 1$ pandemic influenza virus infection in a murine model. Antiviral Res. 89(1), 124-126 (2011).

8 Judy BM, Taylor K, Deeraksa A et al. Prophylactic application of $\mathrm{CpG}$ oligonucleotides augments the early host response and confers protection in acute melioidosis. PLoS ONE 7(3), e34176 (2012).

9 Juffermans NP, Leemans JC, Florquin $S$ et al. CpG oligodeoxynucleotides enhance host defense during murine tuberculosis. Infect. Immun. 70(1), 147-152 (2002).

10 Amlie-Lefond C, Paz DA, Connelly MP, Huffnagle GB, Whelan NT, Whelan HT. Innate immunity for biodefense: a strategy whose time has come. J. Allergy Clin. Immunol. 116(6), 1334-1342 (2005).

11 National Research Council. Treating Infectious Diseases in a Microbial World: Report of Two Workshops on Novel Antimicrobial Therapeutics. The National Academies Press, DC, USA (2006). 
12 Beutler B. Inferences, questions and possibilities in Toll-like receptor signalling. Nature 430 (6996), 257-263 (2004).

13 Lawton JA, Ghosh P. Novel therapeutic strategies based on toll-like receptor signaling. Curr. Opin. Chem. Biol. 7(4), 446-451 (2003).

14 Salata O. Applications of nanoparticles in biology and medicine. J. Nanobiotechnol. 2(1), 3 (2004).

15 Cho K, Wang X, Nie S, Chen ZG, Shin DM. Therapeutic nanoparticles for drug delivery in cancer. Clin. Cancer Res. 14(5), 1310-1316 (2008).

16 Schuler MA, Denisov IG, Sligar SG. Nanodiscs as a new tool to examine lipid-protein interactions. Methods Mol. Biol. 974, 415-433 (2013).

17 Fischer NO, Rasley A, Corzett M, Hwang MH, Hoeprich $\mathrm{PD}$, Blanchette CD. Colocalized delivery of adjuvant and antigen using nanolipoprotein particles enhances the immune response to recombinant antigens. J. Am. Chem. Soc. 135(6), 2044-2047 (2013).

18 Frias JC, Ma Y, Williams KJ, Fayad ZA, Fisher EA.

Properties of a versatile nanoparticle platform contrast agent to image and characterize atherosclerotic plaques by magnetic resonance imaging. Nano Lett. 6(10), 2220-2224 (2006).

19 Nelson KG, Bishop JV, Ryan RO, Titus R. Nanodiskassociated amphotericin B clears Leishmania major cutaneous infection in susceptible BALB/c mice. Antimicrob. Agent. Chemother. 50(4), 1238-1244 (2006).

20 Burgess BL, He Y, Baker MM et al. NanoDisk containing super aggregated amphotericin B: a high therapeutic index antifungal formulation with enhanced potency. Int J. Nanomed. 8, 4733-4743 (2013) 